\title{
Commissioning of the New sMDT Monitored Drift Tube Detectors for the Phase-1 Upgrade of the ATLAS Muon Spectrometer
}

\author{
E. Voevodina* \\ (on behalf of MPI Munich sMDT group) \\ Max-Planck-Institute for Physics, Munich, German \\ Received December 23, 2020; revised December 23, 2020; accepted December 23, 2020
}

\begin{abstract}
The Muon Drift Tube (MDT) chambers provide very precise and reliable muon tracking and momentum measurement in the ATLAS muon spectrometer. Already in Run 2 of the LHC they have to cope with very high background counting rates up to $500 \mathrm{~Hz} / \mathrm{cm}^{2}$ in the inner endcap layers. At HighLuminosity LHC (HL-LHC), the background rates are expected to increase by almost a factor of 10 . New small (15 mm)-diameter Muon Drift Tube (so-called sMDT) detectors have been developed for upgrades of the muon spectrometer. They provide an order of magnitude higher rate capability and allow for the installation of additional new triplet thin-gap RPC trigger chambers in the barrel inner layer of the muon detector for HL-LHC. They have been designed for mass production at the Max Planck Institute (MPI) for Physics in Munich and achieve a sense of wire positioning accuracy of $5 \mu \mathrm{m}$. A pilot project for the barrel inner layer upgrade is underway during the 2019/2021 LHC shutdown. Several sMDT chambers have already been installed and operated in the ATLAS detector. The detailed studies of the muon detection efficiency and muon track resolution have been carried out after the assembling of the sMDT detectors in MPI and repeated at CERN after the integration with the new RPC detectors. The author will describe the detector design, the quality assurance and certification path, as well as will present the status of the installation and commissioning, worth its preliminary results and an overview for the complete integration of the sMDT project In the ATLAS experiment.
\end{abstract}

DOI: $10.1134 / \mathrm{S} 1063778821040281$

1. SMDT DETECTORS

FOR THE ATLAS MUON SPECTROMETER UPGRADE

ATLAS muon spectrometer will undergo major modifications to be able to cope with new conditions while exploiting the physics potential of the HL-LHC. The upgrades of the LHC are divided into two phases: Phase-1 in 2019/2021 and Phase-2 in 2025/2027 [1]. The LHC accelerator complex will increase not only the instantaneous and integrated luminosity, but also will drastically increase the data and trigger rates. In order to cope with the huge data flow while maintaining high muon detection efficiency and reducing fake muons found at Trigger Level-1, in the present ATLAS Endcap region the small wheel muon detector will be replaced by a New Small Wheel (NSW) detector for high luminosity LHC runs. The NSW will consist of two new detector technologies: resistive MicroMegas ( $M M)$ and small strip Thin Gap Chambers (sTGC) [2]. Both detector technologies will improve the online muon identification capa-

*E-mail: elena.voevodina@cern.ch bility by using its precise reconstruction of candidate muon track segments.

A part of the muons triggered in the end-cap system do not traverse the new small wheels, but the end of the innermost barrel detector layers. In order to reject fake muon triggers in this region [3], two new detector technologies, triplet thin-gap Resistive Plate Chambers (RPC) and small-Diameter Muon Drift-tubes (sMDT) detectors, will be installed. This project is named as a Barrel Inner (BI) upgrade: it consists of 276 new RPC and 96 sMDT detectors. Before implementing those amounts of new chambers, during Phase-1, the "BIS78" project will be realized [4]. The first 16 new BIS78 sMDT detectors is currently under installation and commissioning: this pilot project aims to validate the final mechanical installation procedure, the correctness of the services previously installed in the experimental cavern, and to obtained an early feedback about the front-end electronics response and detection performance in the ATLAS environment with the detectors powered and read out through the final services. 


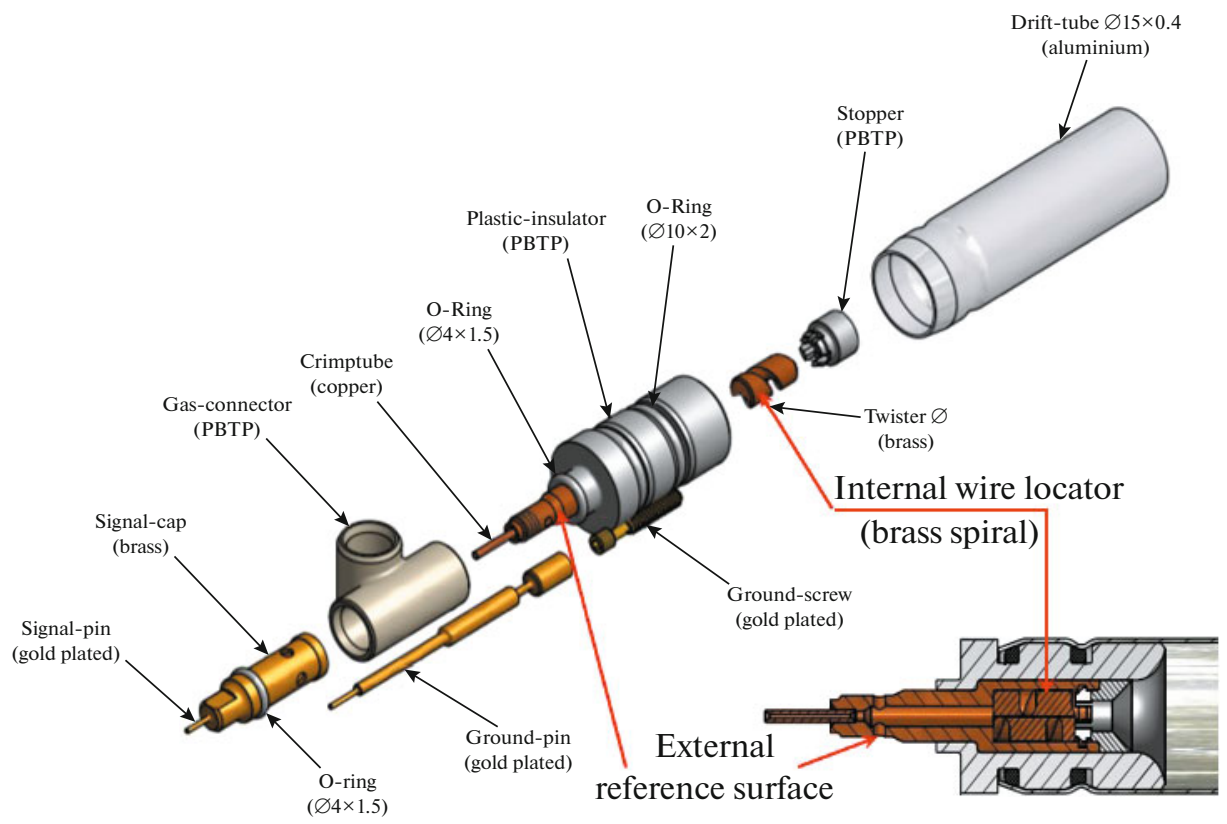

Fig. 1. Exploded view of the sMDT drift tube endplugs with interfaces for precise wire positioning and measurement, for gas and high-voltage supplies, and for read-out electronics.

\section{2. sMDT DETECTORS MECHANICAL DESIGN}

\subsection{Drift Tube Design and Construction}

The major difference between MDT and sMDT detectors is the diameter of the pressurized drift tubes: the individual drift tubes are made of aluminum, have an outer diameter of $15 \mathrm{~mm}$, which is a factor of 2 smaller compared to the current MDT detectors, and a wall thickness of $0.4 \mathrm{~mm}$, which is chromatized in order to ensure reliable electrical ground contacts. A gold-plated W/Re (97/3) anode wire, with a diameter of $50 \mu \mathrm{m}$, is fixed in the insulating plastic endplugs for centering at both ends of the tubes. The mechanical assembly of the tubes is carried out by means of a semi-automated wiring station in a climatized clean room. The wires are inserted into the tubes and endplugs using clean air flow, fixed within crimped copper tubelets in the centres of the endplugs and stretched with a wire tension equivalent to $350 \pm 15 \mathrm{~g}$. The wires are positioned with a precision of a few $\mu \mathrm{m}$ with respect to the cylindrical external reference surface on the central brass insert of the endplugs. The detailed view of the individual tube can be seen in Fig. 1. Additional details and technical aspects of the sMDT detector design and assembly techniques are available in $[4,5]$.

The tubes are operated with an $\mathrm{Ar} / \mathrm{CO}_{2}(93 / 7 \%)$ gas mixture at 3-bar absolute pressure at a gas gain of $2 \times 10^{4}$, which corresponds to an applied high voltage of about $2730 \mathrm{~V}$ between the anode wires and the tube walls. An average single tube resolution of about
$100 \mu \mathrm{m}$ can be achieved and a maximum drift time for electrons from hits near the tube wall to the anode wire is about $190 \mathrm{~ns}$ [4]. Apart from the more compact mechanical design, the diameter reduction leads to a significant decrease of the background occupancy by a factor of eight due to the four times shorter maximum drift time and the two times smaller tube crosssection exposed to the photon and neutron background radiation in the ATLAS environment. The reduction in the drift time also allows for a shortening of the electronics dead time without an increase of hit rate and output bandwidth. Standardized assembly and quality control procedures have been established in order to prevent any mechanical or electrical issues that might affect the detector performance, by measurements of the wire tension, the dark current under high voltage and the gas leakage rate. The latter is required to be less than $0.23 \mathrm{mbar} / \mathrm{h}$ for tubes of 1-m length: an overall failure rate of only $2 \%$ has been achieved. Failures are mostly due to dark currents greater than $2 \mathrm{nA} / \mathrm{m}$, which could be caused by imperfections of the inner surface of the tubes. An average tube production rate of 60 tubes per day is achieved with three technicians operating one assembly line, one wire tensioning station and one high voltage and gas leakage stand tests.

\subsection{Detector Design and Construction}

The sMDT detectors consist of two multilayers of drift tubes, each containing four layers of densely packed drift tubes mounted on an aluminum support 

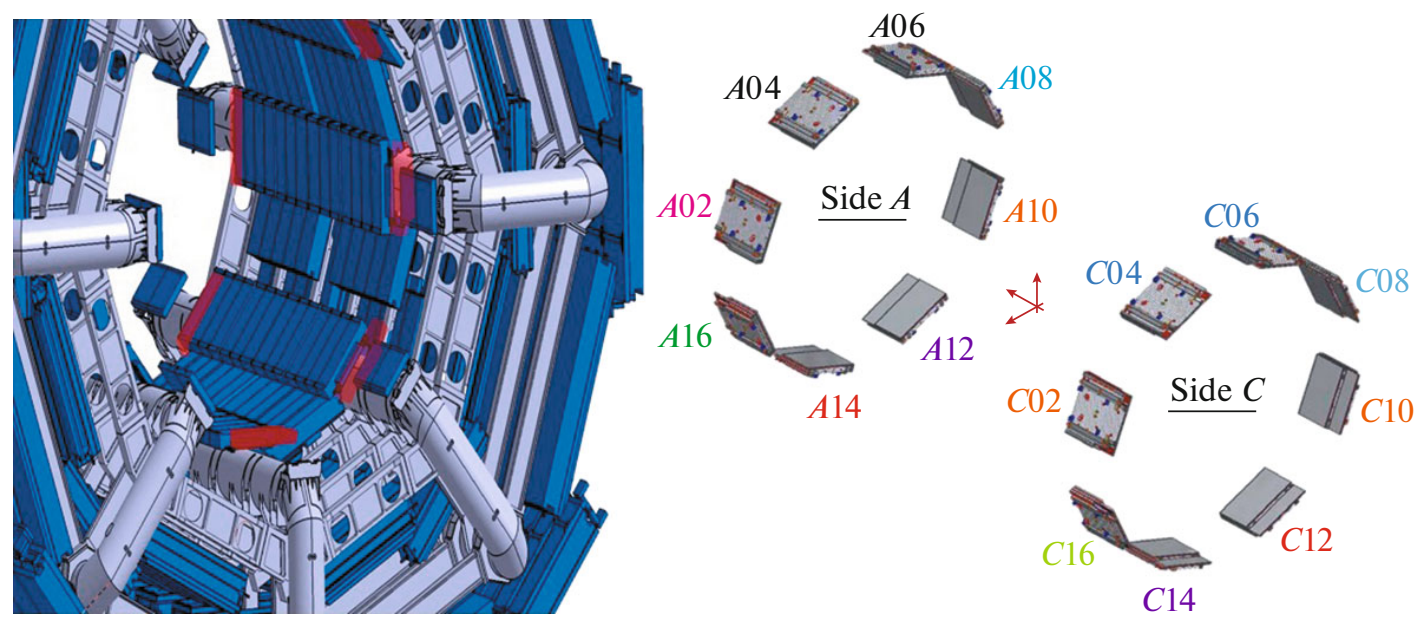

Fig. 2. The ATLAS muon spectrometer barrel with the new BIS78 muon stations. Drawing of the 12 different $A / C$ side sMDT detectors versions.

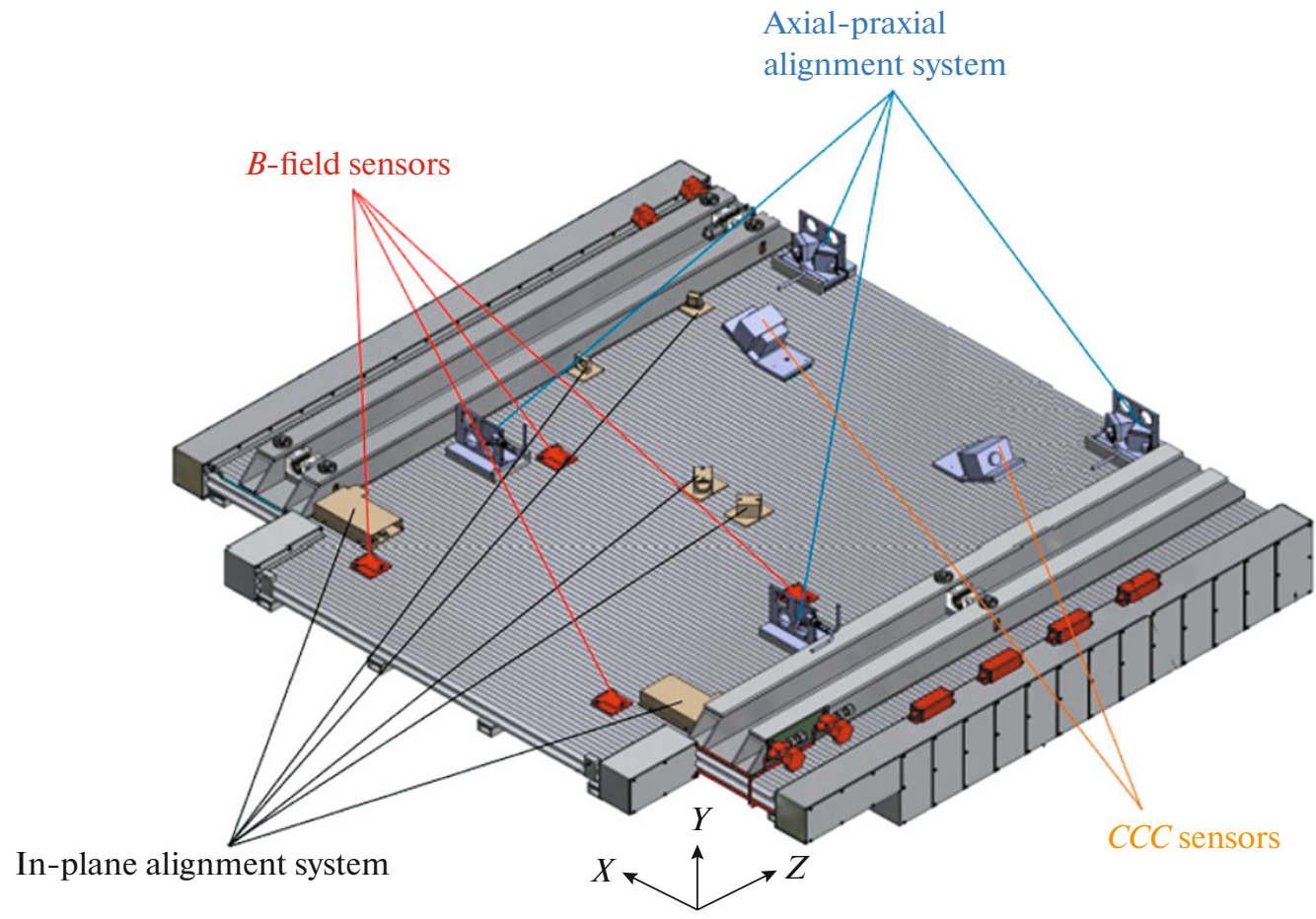

Fig. 3. 3D-drawing of the BIS78 sMDT detector with in-plane, barrel alignment and $B$-field sensors mounted on platforms on the top chamber multilayer.

frame: the chamber sizes vary from about 2.67 to $3.04 \mathrm{~m}^{2}$ while tube lengths range from 1009 to 1669 $\mathrm{mm}$. In order to uniformly fill the space by taking into account the features of the toroid magnet coils at the ends of the barrel inner layer and to reduce the number of dead areas, 12 different sMDT detectors versions have been properly designed [6] (Fig. 2). For detector control data, the BIS78 sMDT chambers are equipped with 12 temperature sensors distributed on the multilayers and the support structure. On the top surface of the detector are typically mounted eight platforms: four Axial/Praxial, two CCC and two $B$-field platforms. In addition, each detector is instrumented with the in-plane alignment system, consisting of two image sensors, four lenses, and two or four masks, in order to monitor the deformations such as sag and torsion (Fig. 3). For the $16 \mathrm{~A} / \mathrm{C}$-side BIS78 sMDT detectors, the chamber-to-chamber alignment system and $B$-field components will be reused from the previous old MDT detectors.

Before and after the detector assembly, a strict quality control protocol is carried out on all detector 


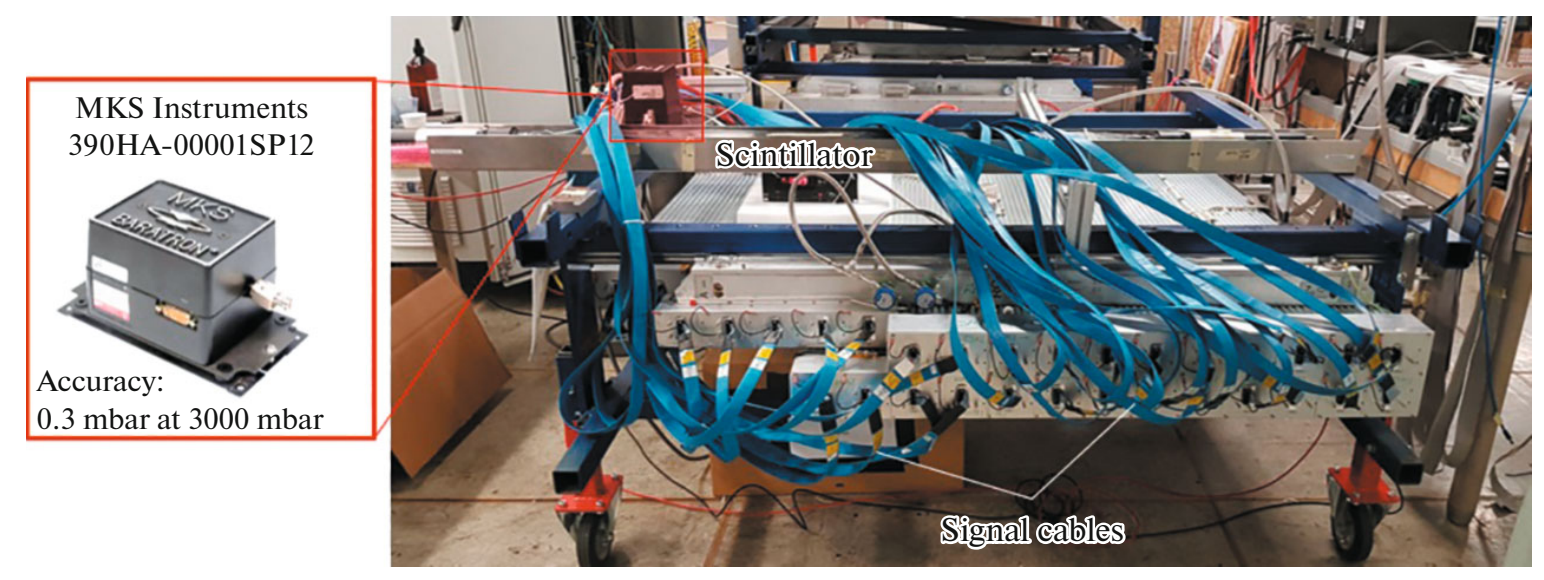

Fig. 4. Picture of one of the sMDT detectors during the surface commissioning test campaign at CERN "BB5" facility.

components as well as on assembled detectors to ensure that the material, chamber components, as well as the fully assembled detector meet different acceptance criteria and requirements before the final installation into the ATLAS experiment.

16 new sMDT chambers have been successfully assembled and fully tested and commissioned at the MPI production site [7] in the time period between 2017 and 2019. At the beginning of June 2020, they have been delivered from MPI-Munich to CERN "BB5" construction facility.

\section{VALIDATION TESTS AND INTEGRATION OF THE SMDT DETECTORS}

Upon their arrival at CERN "BB5" construction facility, the sMDT detectors are subjected to a validation test program: gas tightness, connectivity and the noise test of the front-end electronics and test with cosmic ray, in order to discover any damage which might have occurred during the transport from the MPI production site and to ensure their proper operation in the ATLAS muon spectrometer.

\subsection{Gas Tightness}

To detect the gas leakage, each detector is filled with the nominal $\mathrm{Ar} / \mathrm{CO}_{2}(93 / 7 \%)$ gas mixture to the operating absolute pressure of 3 bar. The pressure values are read by using a Baratron heated reference differential pressure gauge, while the temperature values are read out by using 12 sensors glued to the top surface of the sMDT detector. The gas valves are closed to determine the pressure loss of the chamber over a time period of at least $24 \mathrm{~h}$. A picture of the sMDT detector under test is shown in Fig. 4.

\subsection{Connectivity and Noise Test of the Front-End Electronics}

The goal of the connectivity test is to check the proper connectivity of the electronics components, calibrate the front-end electronics parameters, as well as to check the communication stability. The noise measurement at the operating high voltage of $2730 \mathrm{~V}$ and the threshold value of $-39 \mathrm{mV}$ is carried out by measuring the dark current of each tube layer. The noise rate is determined by randomly triggering the front-end electronics and scaling the number of hits by the inverse of the integral active time window of the data acquisition. The test is performed at both detector configurations: without and with applied high voltage, thus the electronic noise and discharges occurring inside the drift tubes can be distinguished. The average intrinsic noise rate per tube for a single chamber service module (CSM) is presented in Fig. 5. The results were obtained by excluding individual noisy tubes which are satisfied the following condition:

$$
\text { Noise }_{i}^{\mathrm{ON}}>5 \mathrm{kHz} \wedge \text { Noise }_{i}^{\mathrm{ON}}>2 \times \text { Noise }_{i}^{\mathrm{OFF}},
$$

where $\mathrm{Noise}_{i}^{\mathrm{ON}}$ and Noise ${ }_{i}^{\mathrm{OFF}}$ correspond to the determined noise levels of the $i$ th tube in a detector with and without applied high voltage, respectively.

\subsection{Cosmic Ray Test}

In the final phase of the validation campaign, all BIS 78 sMTD detectors have to be tested with cosmic ray muons in order to determine the spatial resolution and muon detection efficiency. It allows to crosscheck that the drift tube recorded hit if a reconstructed track is passing through the tube. The measurements are performed at the nominal operating high voltage and threshold values. Data is taken separately for both chamber service modules with the same data taking time of about $15 \mathrm{~h}$. The muon tracks are 
Average intrinsic noise rate, $\mathrm{kHz} /$ tube

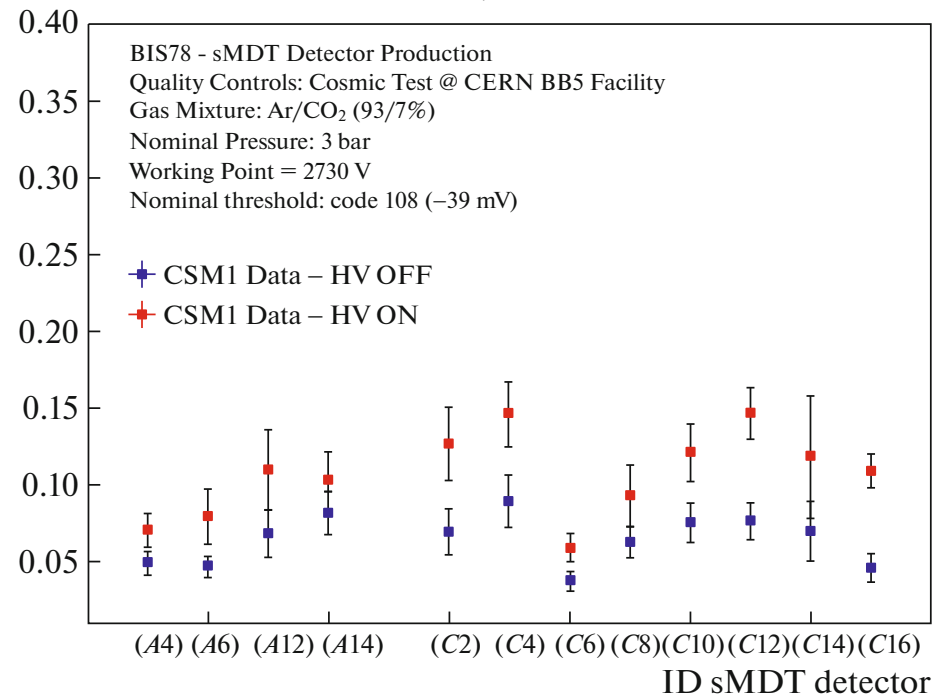

Fig. 5. Average intrinsic noise rate per tube/channel for one chamber service module of each tested BIS78 sMDT detectors at the operating high voltage point of $2730 \mathrm{~V}$ and nominal threshold of $-39 \mathrm{mV}$.

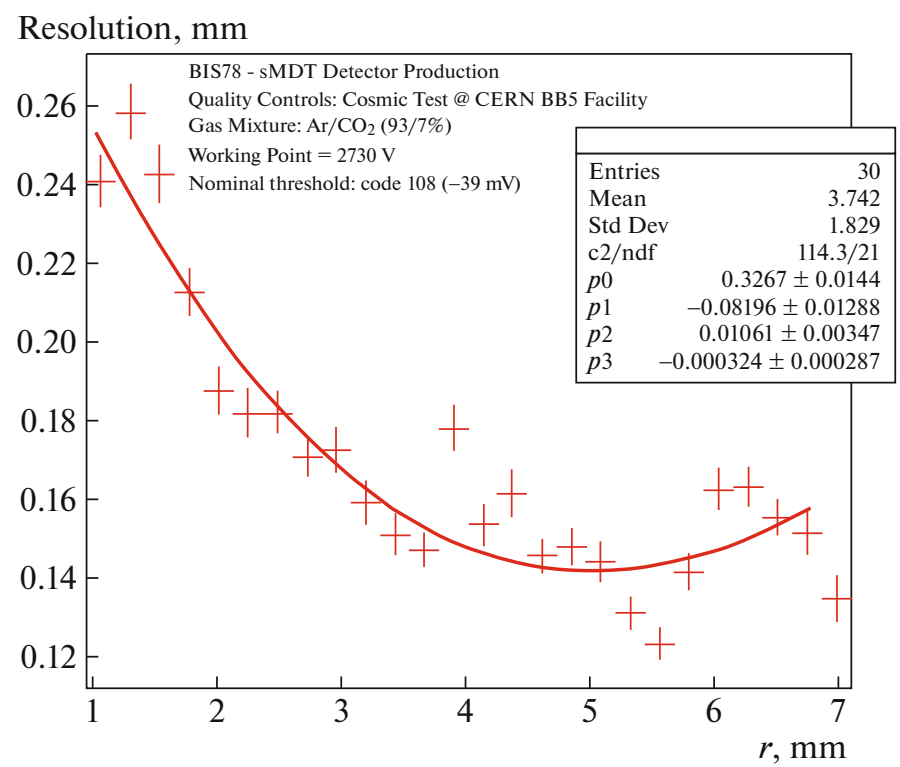

Fig. 6. Spatial resolution as a function of the measured drift radius $(r)$ of the tested $A 8$ chamber at the operating high voltage point of $2730 \mathrm{~V}$ and nominal threshold of $-39 \mathrm{mV}$. The result is presented for the one chamber service module (CSM 1 ).

reconstructed by requiring mimimum of four hits in the detector. As an example, the result for the dependency of the spatial resolution of the drift radius for one tested BIS78 $A$ side sMDT chamber is shown in Fig. 6. The presented data are before subtracting the contribution of the multi scattering, obtained from Monte Carlo simulation.

Due to the strong energy dependence of the cosmic ray muon flux $j \sim E^{-2}$ most of the cosmic muons are of low momentum $p_{\mu}<1 \mathrm{GeV} \mathrm{c}^{-1}$ and affected by multiple scattering in the detector. The multiple scattering contributes significantly to the spatial resolution determined using cosmic muon tracks:

$$
\sigma_{\text {mean }}=\sqrt{\sigma_{\text {tube }}^{2}+\sigma_{\text {MS }}^{2}},
$$

where $\sigma_{\text {tube }}$ is the tube spatial resolution, $\sigma_{M S}$ is the contribution of the multiple scaterring. By taking into account the results obtained by the detector simulation in Geant4, $\sigma_{M S}=120 \pm 10 \mu \mathrm{m}$ for the cosmic muon momentum spectrum in the test stand [8]. Moreover, $\sigma_{\text {tube }}$ is taken from the high energy muon test beam measurements. 
Spatial resolution, $\mu \mathrm{m}$

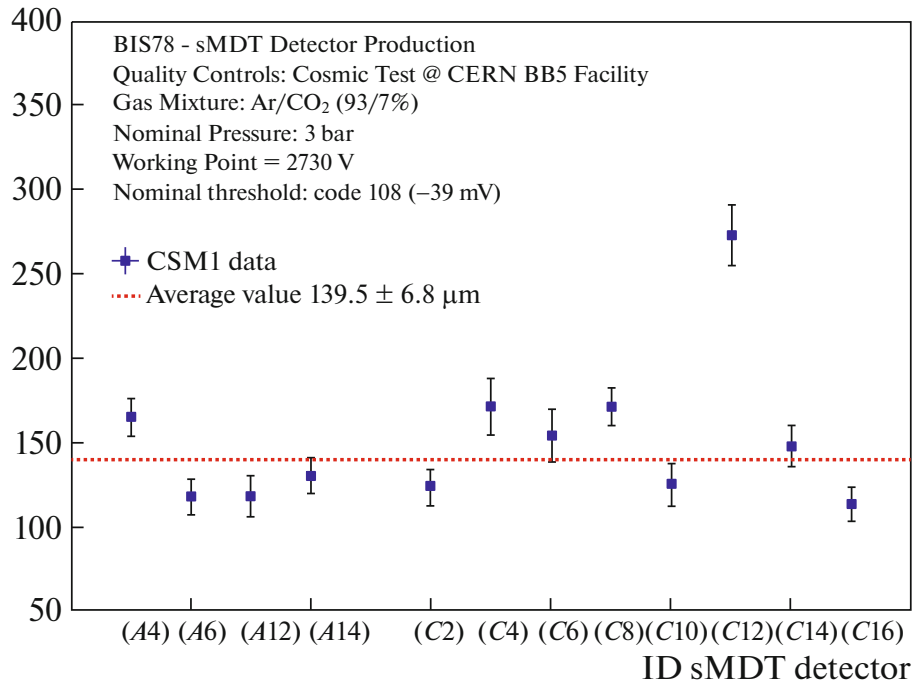

Fig. 7. Drift tube spatial resolution results of all BIS78 sMDT detectors at the operating conditions.

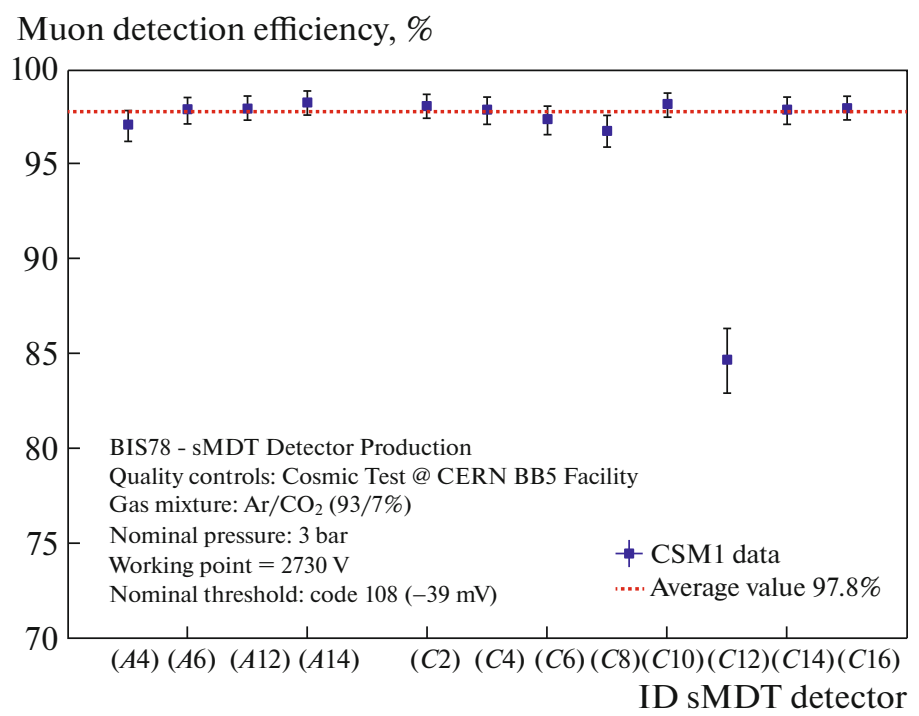

Fig. 8. Average drift tube muon detection efficiency of the twelve tested BIS 78 sMDT detectors at the operating conditions.

Figure 7 shows the mean tube resolution in each tested BIS78 sMDT detector by including the contribution from multiple scattering. The average detector spatial resolution is $142.2 \mu \mathrm{m}$ which is in agreement with the expectation from the test beam measurement with high energy muons [9].

The muon detection efficiency has been also determined by using the cosmic ray data. The reconstructed tracks are extrapolated to each tube layer. The tube hit efficiency is measured as the probability to detect a hit in the tube that the muon passed through. The muon tracks are reconstructed by requiring a minimum of four hits. The single tube efficiency is computed as:

$$
\varepsilon_{\text {tube }, \mu}=\frac{N_{\text {hits }}}{N_{\text {tracks }}},
$$

where $N_{\text {hits }}$ is the number of the detected hits in tube matching the reconstructed tracks and $N_{\text {tracks }}$ is the number of all reconstructed tracks crossing the tube. The final measured average tube muon detection efficiency of all sMDT detectors is presented in Fig. 8. The average muon efficiency result of all sMDT detectors is about $97.2 \% \pm 0.2 \%$ which is in agreement with the ATLAS requirements [4].

The lower efficiency result of the $C 12$ detector is due to the high average intrinsic noise rate of the 

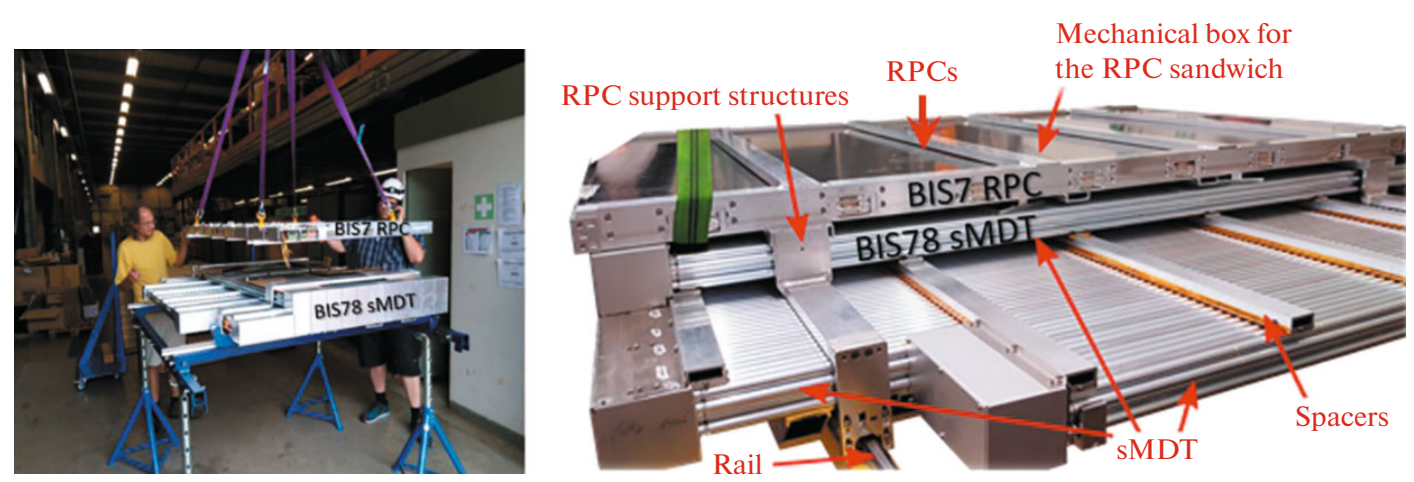

Fig. 9. The part of the mechanical integration procedure of a BIS78 sMDT and BIS7 RPC detectors. The three thin gas gaps of the RPC detector are mounted inside an aluminum frame which holds them together and limits gravitational sag. As in the ATLAS experiment cavern, the triplet RPC and the accompanying sMDT detectors are mounted on rails with independent mechanical supports.

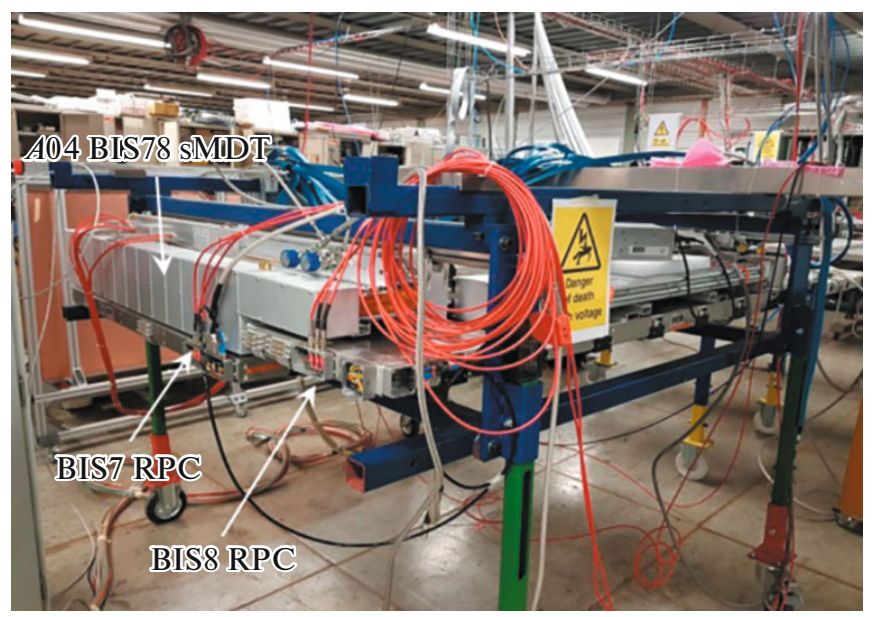

Fig. 10. The final BIS78 muon station is composed of the sMDT detector in the upper part and the triplet BIS7 and BIS8 RPC chambers in the lower part. The muon module is under the last interference test at CERN "BB5" construction facility.

Average intrinsic noise rate, $\mathrm{kHz} /$ tube

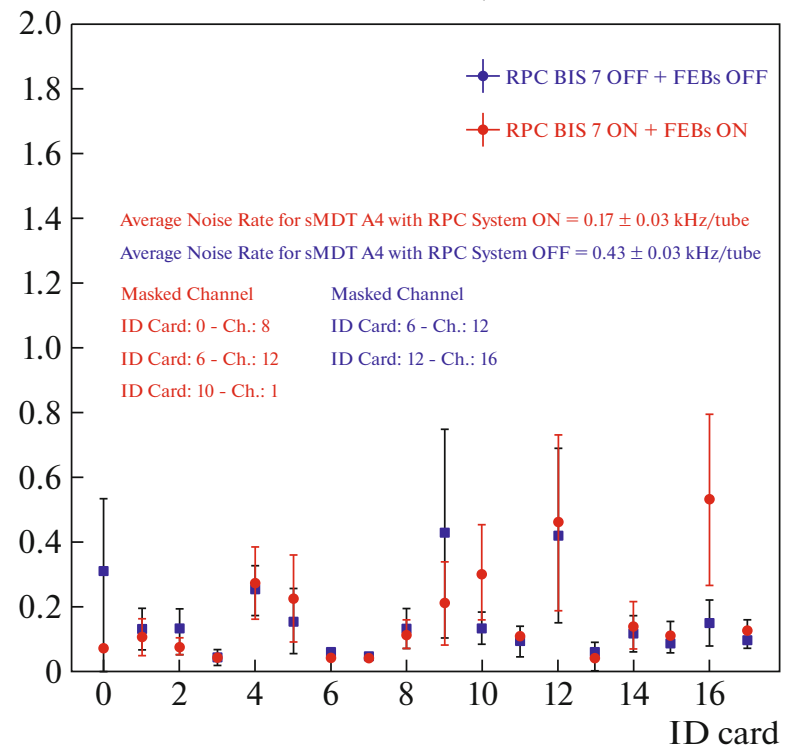

Fig. 11. Example of the sMDT chamber interference test result obtained for one chamber service module. front-end electronics. The noise problem of the $C 12$ detector is in-depth investigation.

\section{CHAMBERS INTEGRATION}

After the initial tests, the sMDT detector is then integrated with its two new triplet BIS7 and BIS8 RPC trigger detectors to form the muon station. The BIS muon stations require a special common support frame to carry the RPC and the sMDT detectors, as shown in Fig. 9, due to their position on the inner region of the ATLAS toroid magnet coils. The RPCs are inserted in the support frame and the sMDT detector is mounted on top by using the kinematical bearings. After chamber integration procedures the BIS78 sMDT detector should pass the interference test at the two BIS78 RPC configurations: with and without applied high voltage. The BIS78 muon module under interference test is presented in Fig. 10. As shown in Fig. 11, the effect of the RPC detectors and their electronics is negligible to the sMDT detector system in the term of electrical noise. 

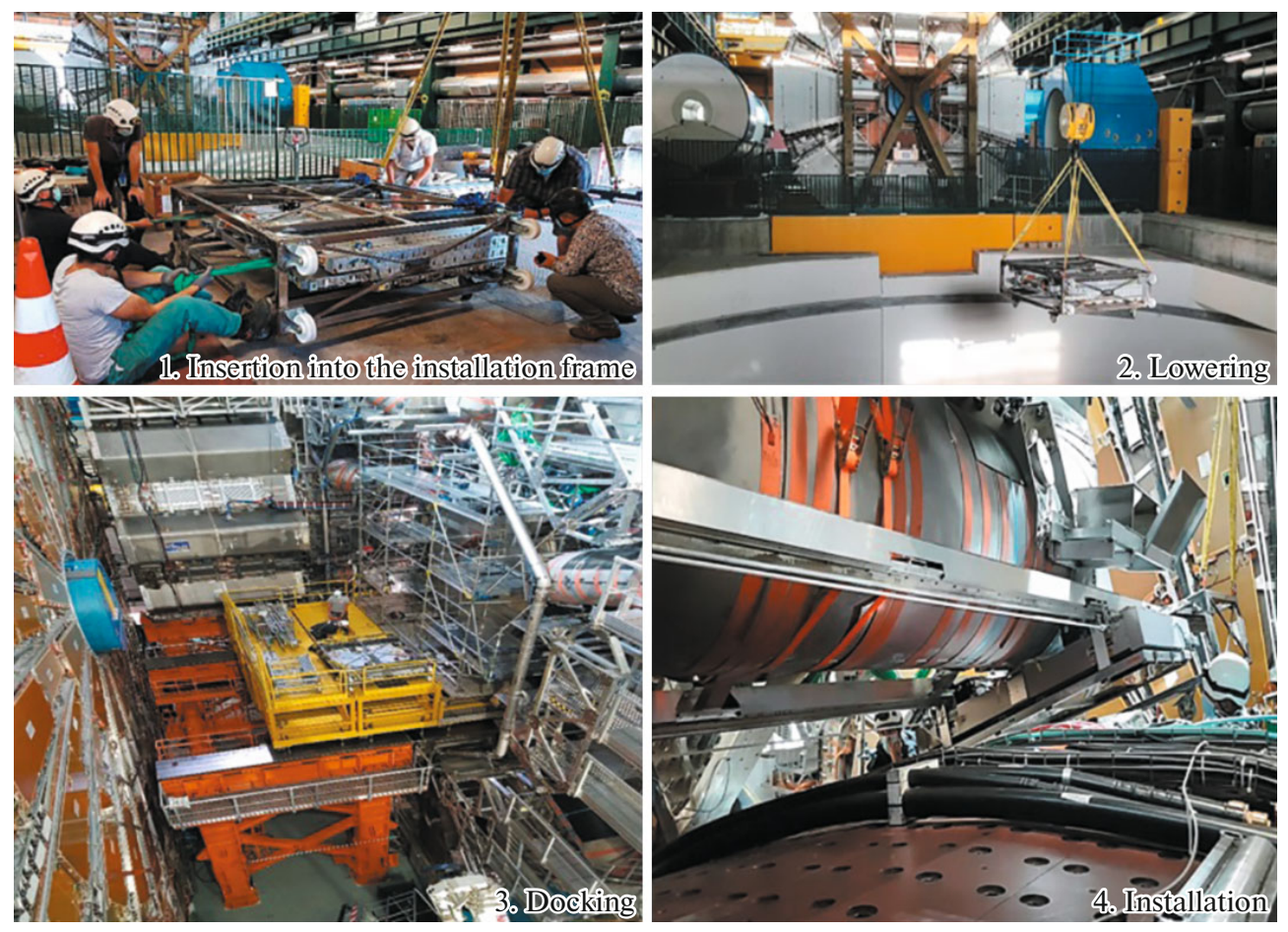

Fig. 12. Pictures of the main installation procedures obtained during the first BIS78 muon station installation in sector $A 4$ of the inner barrel region of the ATLAS muon spectrometer.

After the interference test campaign, the sMDT detector is equipped with $B$-field and Axial-Praxial, CCC alignment sensors whose proper operation is then verified.

\section{INSTALLATION AND COMMISSIONING OF THE SMDT DETECTORS IN THE ATLAS MUON SPECTROMETER}

At the surface above the ATLAS experimental cavern, the mechanical integrity of the station is checked to ensure that no damage occurred during transport from the integration site. The new muon station is placed into an installation frame and lowered into the underground experimental hall. There, the installation frame is supported by two cranes, rotated to the appropriate angle, and docked to the rail system of the ATLAS Muon Spectrometer. The muon station is slid onto the rails by using the two winches, positioned with an accuracy of about a few $\mathrm{mm}$ in all three spatial directions and fixed at one bearing on the rail. All installation procedures of the first BIS78 muon station in the sector A04 of the ATLAS muon spectrometer is shown in Fig. 12. The service lines at ATLAS cavern: gas distribution, low and high voltage cables, detector control system cables, and read-out fibers, etc. for both sub-module have been carefully cross-checked, routed, and then connected to the muon stations.
With the installation complete, the next step is to fully commission the new muon stations: each installed detector will be tested afterward using the final services together with the Detector Control System, Data Acquisition and Data Quality Monitoring Systems, in preparation for the further cosmic ray data taking, which is a unique opportunity to commission the functionalities and verify the detection performances, and finally to move to the future physics data taking campaigns.

\section{CONCLUSIONS}

During next year, the ATLAS Muon Spectrometer will go through a series of upgrades in order to cope with the foreseen increasing of LHC performance. New BIS78 Detector Module (the triplet thin-gap resistive plate chamber and small-diameter muon drift tube detector) have been constructed for the Phase-1 Upgrade as a pilot project for the ATLAS barrel muon spectrometer in order to increase the trigger acceptance and to prepare for the high background rate requirements of the HL-LHC.

The Max-Planck-Institut fur Physik in Munich (MPI) has built $16 \mathrm{~A} / \mathrm{C}$ side new BIS78 sMDT for the ATLAS barrel muon spectrometer. The detectors have been passed the quality control/quality insurance tests at MPI and send to CERN "BB5" construction and assembly facility in the time period 
2019/2020. Upon their arrival at CERN, all sMDTs were subjected to a test program-gas tightness, connectivity and noise rate tests of the readout electronics, test with cosmic ray-to discover any damage which might have occurred during the transport from the production site and to ensure their proper operation in the experiment. The noise rates were determined per drift tube of the chamber and were found to be in the agreement with previously measured intrinsic noise levels of each channel on the read-out cards obtained at the MPI validation test. The spatial resolution of the tubes in a chamber was computed by using the method that considers almost vertical cosmic muon tracks. Measured values were found to be in agreement with the Monte Carlo prediction and the expectations from previous test beam measurements. The muon detection efficiency was evaluated for each tube in a detector, showing full efficiency and uniform performance of the tested chambers. Obtained results are in agreement with ATLAS physics requirements. Thus, all tested chambers show an uniform response and a consistent geometry, well within the allowed limits. Every sMDT detector that passed the acceptance tests was integrated with their two BIS7 and BIS8 RPC trigger chambers.

The installation and commissioning phases have been fully resumed after the lockdown imposed by the SARS COV-2 health crisis: 4 out of 16 BIS78 sMDT detectors have been successfully installed in the ATLAS experiment. After the connection of the final services, the stations will be fully commissioned with cosmic rays.

\section{REFERENCES}

1. ATLAS Collab., CERN-LHCC-2013-006, CERN (Geneva, 2013).

2. G. Apollinari et al., Tech. Design Report V. 0.1, CERN2017-007-M, CERN (Geneva, 2017).

3. Sh. Akatsuka (on behalf of ATLAS Collab.), in Proceedings of the 21st IEEE Real Time Conference, Williamsburg, Va, USA, 9-15 June, 2018, ATLDAQ-PROC-2018-007.

4. ATLAS Collab., CERN-LHCC-2017-017; ATLASTDR-026.

5. H. Kroha, Nucl. Instrum. Methods A 936, 445 (2019); https://doi.org/10.1016/j.nima.2018.10.139

6. H. Kroha, sMDT Chamber Parameter Book, Version 1-3, Max-Planck-Institut fur Physik (Munich 2017; Oct. 2020).

7. S. Hadzic, Master Thesis, CERN-THESIS-2019150, Tecnical University Munich (2019).

8. O. Kortner, ATL-MUON-2000-021.

9. P. Schwegler, CERN-THESIS-2014-091. 